\title{
Pitiriasis versicolor variedad vitiligoide e intertrigo por Malassezia. Comunicación de un caso
}

\author{
Pityriasis versicolor vitiligoid variety and Malassezia \\ intertrigo. Communication of a case
}

María del Carmen Padilla-Desgarennes, ${ }^{*}$ Mario Rodríguez-Y Silva, ${ }^{\ddagger}$ Karen Lizette Cerda Contreras ${ }^{\S}$

\section{RESUMEN}

La pitiriasis versicolor es una micosis superficial causada por levaduras del género Malassezia en su fase micelial; en su forma de levadura constituye parte de la microbiota cutánea y requiere de factores predisponentes 0 desencadenantes para causar la enfermedad. Existen varias formas clínicas de esta micosis, la más frecuente es la hipocrómica, seguida por la hipercrómica y eritematosa. En este artículo se comunica una variedad clínica infrecuente de pitiriasis versicolor denominada vitiligoide 0 acrómica en un hombre de 19 años. El interés de esta comunicación radica en el diagnóstico diferencial con otras dermatosis hipo o acrómicas, en especial con el vitíligo.

Palabras clave: Pitiriasis versicolor vitiligoide, Malassezia, vitíligo.

\section{ABSTRACT}

Pityriasis versicolor is a superficial mycosis caused by yeasts of the Malassezia genus in their mycelial phase; in its yeast form, it constitutes part of the skin microbiota and requires predisposing or triggering factors to cause the disease. There are several clinical forms of this mycosis, the most frequent is the hypochromic variety, followed by the hyperchromic and erythematous. This article reports an uncommon clinical variety of pityriasis versicolor called vitiligoid or achromic in a 19-year-old man. The interest of this communication lies in the differential diagnosis with other hypo or achromic dermatoses, especially with vitiligo.

Keywords: Pityriasis versicolor vitiligoid, Malassezia, vitiligo.

\section{INTRODUCCIÓN}

La pitiriasis versicolor (PV) es una micosis superficial causada por levaduras del género Malassezia, principalmente M. globosa. Hasta la fecha se han descrito 18 especies de este hongo mediante métodos morfológicos, bioquímicos, fisiológicos y moleculares, de las cuales $M$. furfur sensu stricto, $M$. sympodialis, $M$. globosa, $M$. obtusa, $M$. restricta y $M$. slooffiae se han identificado como agentes causales de esta micosis y de manera excepcional, M. pachydermatis. ${ }^{1-7}$

\section{EPIDEMIOLOGÍA}

Su prevalencia es de hasta $50 \%$ en regiones tropicales, en contraste con $1.1 \%$ en climas fríos. ${ }^{8-10}$ En la Ciudad de México, en el Centro Dermatológico Pascua, representa $8.3 \%$ de las micosis superficiales, ${ }^{8}$ es más común en adolescentes y adultos jóvenes del sexo masculino. La variedad clínica hipocrómica es la más frecuente, seguida de la hipercrómica y eritematosa. A las formas combinadas (hipocrómica más hipercrómica) les corresponde $7.1 \% .{ }^{8}$ En países

\footnotetext{
* Dermatóloga y Micóloga. Jefa del Servicio de Micología.

‡ Dermatólogo egresado.

$\S$ Residente del segundo año de Dermatología.
}

Centro Dermatológico «Dr. Ladislao de la Pascua», SSCDMX.

Citar como: Padilla-Desgarennes MC, Rodríguez-Y Silva M, Cerda CKL. Pitiriasis versicolor variedad vitiligoide e intertrigo por Malassezia. Comunicación de un caso. Rev Cent Dermatol Pascua. 2021; 30 (3): 161-165. https://dx.doi.org/10.35366/103831 
Padilla-Desgarennes MC y cols. Pitiriasis versicolor variedad vitiligoide e intertrigo por Malassezia

tropicales puede oscilar entre 5 y $7 \%$ en menores de 13 años. ${ }^{5,6,8}$

\section{ETIOPATOGENIA}

La PV es ocasionada por hongos de la división Basidiomycota del género Malassezia, estas levaduras son dimórficas y lipofílicas (con excepción de M. pachydermatis). Forman parte de la microbiota cutánea de las zonas seborreicas de la piel y los folículos pilosos. ${ }^{1,11,12}$ Hasta la fecha se han identificado 18 especies del género Malassezia: $M$. furfur, $M$. pachydermatis, $M$. sympodialis, $M$. globosa, $M$. slooffiae, $M$. restricta, $M$. obtusa, $M$. dermatis, $M$. japonica, $M$. nana, $M$. yamatoensis, M. caprae, M. equina, M. cuniculi, M. psittaci, M. brasiliensis, $M$. arunalokei y $M$. vespertilionis. ${ }^{1-4,13}$

Sólo algunas se han identificado como causantes de PV. Malassezia globosa es la más frecuente a nivel mundial, hasta $97 \%$ de los casos, ${ }^{14}$ seguida por M. sympodialis, $M$. slooffiae, $M$. restricta y $M$. furfur. En general se presentan combinadas dos o más. Es probable que el factor geográfico influya en el desarrollo de una u otra especie, ya que en un estudio realizado en Japón se identificó a $M$. furfur y a $M$. sympodialis como los principales agentes causales y en otro trabajo realizado en Argentina, M. sympodialis fue la más frecuente. ${ }^{11,15,16}$

\section{PATOGENIA}

En su fase de levadura, Malassezia sp. se encuentra como saprobio en zonas con gran cantidad de glándulas sebáceas, tales como la piel cabelluda, la cara, el conducto auditivo externo, el tórax, anterior y posterior. Al parasitar la capa córnea de la piel, se introduce también en los folículos pilosebáceos, que actúan como reservorios, lo que puede explicar las recidivas. ${ }^{2,5,8}$

Para que se desarrolle el cuadro clínico de PV es necesario que se transforme a su fase micelial (patógena). Este cambio se produce si se dan condiciones predisponentes favorables: factores exógenos y endógenos.

Entre los factores endógenos se encuentran la predisposición genética, piel seborreica, hiperhidrosis, desnutrición y estados de inmunosupresión, tales como la diabetes, y en pacientes receptores de trasplante de órganos. ${ }^{12,14}$ Algunos autores han detectado alteraciones en la respuesta humoral en pacientes con PV, con aumento en la producción de IgG y defecto en la producción de linfocinas, con disminución de las células T reactivas en sangre periférica. ${ }^{17}$

Entre los factores exógenos se ha mencionado la exposición a humedad ambiental excesiva, realizar actividades deportivas al aire libre, así como uso frecuente de corticoides tópicos y sistémicos. Al parecer, la elevada frecuencia de PV en países tropicales puede ser por la disminución en el recambio celular favorecido por el calor, es probable que la exposición a rayos UVA conduzca a la formación de ácidos grasos hidroxilados que actúen como sustratos para el crecimiento de la levadura. La oclusión por el uso de ropa con alto contenido de fibras sintéticas, la aplicación de aceites y los bronceadores incrementan la producción de $\mathrm{CO}_{2}$, con modificaciones subsecuentes en el pH cutáneo, y alteraciones en la microbiota cutánea, que conducen a mayor desarrollo de Malassezia. ${ }^{17}$

Una vez que los micelios se ubican en el estrato córneo, producen ácido dicarboxílico (ácido azelaico), que actúa mediante inhibición de la dopa-tirosinasa, lo que bloquea la síntesis de melanina y por consiguiente induce el desarrollo de la hipocromía. También se ha propuesto un efecto citotóxico directo sobre los melanocitos, con reducción en el número y tamaño de los melanosomas, lo que explica la discromía en la variedad hipocromiante. ${ }^{7,8,11}$

Se postulan dos teorías sobre la patogénesis de la hiperpigmentación en la PV: la primera se basa en el aumento de espesor de la capa córnea en individuos de piel obscura, la segunda propone en estos individuos la existencia de infiltrado inflamatorio más denso, el cual actúa como estímulo hacia los melanocitos, que resulta en aumento en la producción de melanina. La descamación es consecuencia de la actividad queratolítica del hongo. ${ }^{10,17}$

No son claros los mecanismos mediante los cuales Malassezia evade la respuesta inmune, aunque es probable que se deba a los mananos y lípidos de su pared. Se ha documentado que puede activar el complemento tanto por vía alterna como por vía directa, y que esto puede estimular el desarrollo de la inflamación. ${ }^{17}$

\section{CUADRO CLÍNICO}

Las lesiones de PV se ubican en el tórax hasta en el $96 \%$ de los casos, en el cuello y en las extremidades superiores en el $25 \%$, la topografía menos frecuente corresponde a los pliegues (antecubitales, axilares, inguinales) en el $2 \%$; a esta variedad clínica se le denomina intertrigo por Malassezia. ${ }^{6-8,18}$

La PV se caracteriza por la presencia de manchas con escama fina en su superficie, que confluyen formando placas de color, forma y tamaño variable, con lo que se establece la clasificación clínica. ${ }^{6-8}$

Las variantes hipocrómica, hipercrómica, eritematosa, vitiligoide y dermatofitoide ${ }^{8}$ en general son asintomáticas, y de forma excepcional los pacientes refieren prurito leve. 
La PV variedad vitiligoide, acrómica, acromiante o «alba» se caracteriza por la presencia de placas acrómicas que semejan vitíligo, cuya superficie está cubierta por escama furfurácea tan fina que es difícil verla a simple vista. ${ }^{6,19,20}$

\section{DIAGNÓSTICO DIFERENCIAL}

En la PV vitiligoide el motivo principal de la consulta es el aspecto cosmético causado por la acromía.

Estas placas son más evidentes en la piel oscura, y se acentúan después de una exposición solar prolongada. ${ }^{21,22}$

El diagnóstico diferencial se realiza con dermatosis que cursan con hipocromía o acromía, tales como: vitíligo, dermatitis solar hipocromiante, pitiriasis alba, hipopigmentación postinflamatoria, hipomelanosis macular progresiva idiopática e hipomelanosis guttata. Otras dermatosis de interés, pero menos frecuentes, son: lepra caso indeterminado, parapsoriasis y micosis fungoide hipopigmentada..$^{7,22}$

Existen reportes en la literatura de casos de PV variedad vitiligoide en topografías inusuales tales como los párpados, prepucio, glande y la areola del pezón, que por su semejanza clínica fueron tratados inicialmente como vitíligo, condicionando un retraso en el diagnóstico. 16,18,21-24

\section{DIAGNÓSTICO}

El diagnóstico es clínico y se puede utilizar la dermatoscopía para observar la escama fina. ${ }^{7,25}$ Como auxiliar, la lámpara de Wood permite apreciar las placas con una fluorescencia amarillo-dorado. .,8 $^{2}$

El estándar de oro es el examen micológico directo con cinta adhesiva y tinción de Albert (azul de toluidina, verde de malaquita, ácido acético glacial, etanol y agua destilada) que facilita la observación de las estructuras fúngicas que se tiñen de color púrpura, filamentos gruesos y cortos en forma de "s cursiva», con levaduras redondas arracimadas, algunas gemantes, características de Malassezia sp..$^{5,8,26}$

Se cultiva en Sabouraud adicionado con $10 \%$ de aceite de oliva, y en el medio Dixon y Dixon modificado. ${ }^{2,8}$ También se utilizan métodos fisiológicos y bioquímicos tales como: reacción de la catalasa y asimilación de Tween. ${ }^{26}$

Los métodos idóneos para la identificación de las especies son los basados en técnicas moleculares, como la reacción en cadena de la polimerasa (PCR) anidada, PCR en tiempo real, electroforesis en gel de campo pulsado (PFGE), amplificación aleatoria de ADN poli- mórfico (RAPD), polimorfismo de longitud de fragmento amplificado (AFLP), electroforesis en gel de gradiente desnaturalizante (DGGE), análisis de ADN ribosómico, análisis ITS (regiones espaciadoras transcritas internas) rADN, análisis IGS (regiones espaciadoras intergénicas) rADN, polimorfismo de longitud de fragmento terminal (tFLP), polimorfismo de longitud de fragmento de restricción (RFLP) y análisis de secuenciación. 4,18

\section{TRATAMIENTO}

El tratamiento tópico debe realizarse indicando derivados azólicos, terbinafina, ciclopiroxolamina y queratolíticos. ${ }^{5}$ Se deben evitar los vehículos oleosos debido a la lipofilia de Malassezia.

En los casos diseminados, con recurrencias frecuentes, son útiles los antifúngicos por vía oral, como el ketoconazol, $200 \mathrm{mg}$ al día durante dos semanas, itraconazol $200 \mathrm{mg}$ al día por una o dos semanas; también se ha utilizado fluconazol $150 \mathrm{mg}$ a la semana durante cuatro semanas. ${ }^{5,7,22}$

Las recidivas son frecuentes en este micosis; esto se debe más bien a la predisposición genética del paciente, así como a la dificultad de evitar los factores predisponentes, tales como una exposición solar excesiva, el uso de cremas, bronceadores en vehículos oleosos, así como el uso de ropa sintética que condiciona hiperhidrosis. En pacientes que migran a climas templados o fríos la micosis desaparece y se reactiva cuando regresan a su lugar de origen. Es de utilidad el uso de jabones con azufre, más ácido salicílico y champú que contengan disulfuro de selenio o piritionato de zinc durante una semana al mes. 6,25

\section{CASO CLÍNICO}

Se comunica el caso de un hombre de 19 años, originario y residente de Cuernavaca, Morelos, quien acude a consulta por presentar «manchas blancas» en los antebrazos que se extendieron al resto del cuerpo, de seis meses de evolución, las cuales le ocasionaban leve prurito en forma ocasional.

Presenta dermatosis diseminada a cuello, tronco y extremidades superiores. Del cuello afecta la cara posterior, de la segunda el tórax anterior y posterior en su tercio superior, con predominio en la región intermamaria, y de la tercera compromete los brazos y antebrazos en sus caras anteriores y posteriores, así como en los pliegues antecubitales. Con tendencia a la simetría (Figura 1). Esta dermatosis estaba constituida por numerosas manchas acrómicas, con escama fina en su superficie, 
en su mayoría circulares, de tamaño variable, entre 1-3 cm, algunas confluían formando placas de mayor tamaño (Figuras 1 y 2). Con estos datos se efectúan los diagnósticos iniciales de hipomelanosis macular progresiva idiopática contra vitíligo contra pitiriasis versicolor variedad vitiligoide, por lo que se solicita estudio micológico directo.

A la observación con lámpara de luz de Wood se aprecia fluorescencia amarillo-dorado, y en el examen directo con tinción de Albert se visualizan abundantes le-

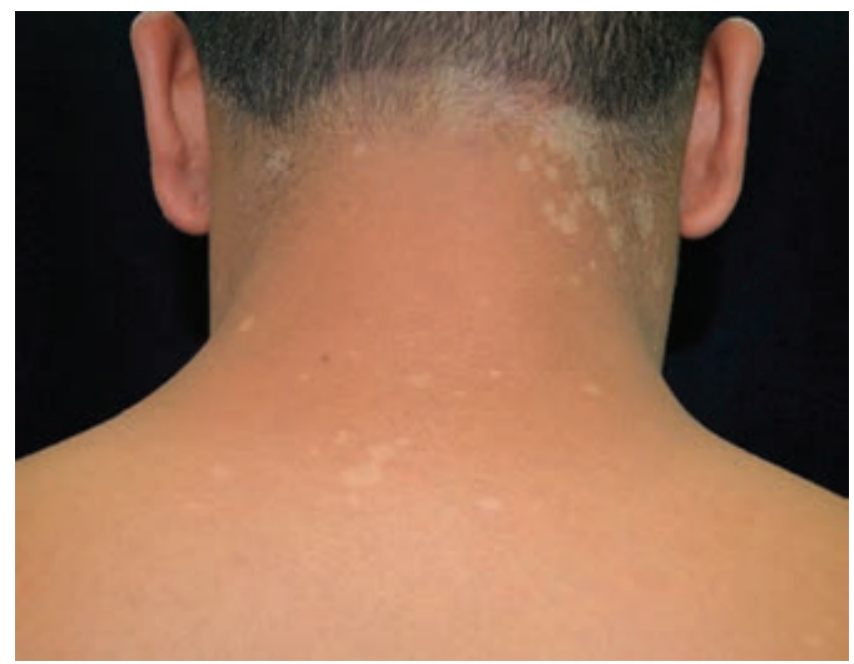

Figura 1: Aspecto clínico. vaduras de color púrpura, grandes, pequeñas, redondas, arracimadas, algunas gemantes, así como filamentos cortos y gruesos (Figura 3). Los hallazgos anteriores fueron compatibles con la parasitación por Malassezia sp., con lo cual se confirma el diagnóstico de pitiriasis versicolor variedad vitiligoide o acrómica por Malassezia $s p$. Se prescribe itraconazol 100 mg vía oral cada 24 horas por dos semanas, terbinafina solución tópica y jabón con ácido salicílico. Tres meses después refiere mejoría significativa, pero persisten placas pequeñas con escama fina en la superficie. Se indica la aplicación de champú con disulfuro de selenio al 2.5\% durante el baño en áreas afectadas. Un mes después el paciente acude por recurrencia de la PV, llama la atención que en ese momento la imagen clínica corresponde a la variedad eritematosa dermatofitoide. Se le explica ampliamente la naturaleza de la micosis, así como la importancia de practicar las medidas generales; se administra itraconazol 200 mg por tres semanas y posteriormente $400 \mathrm{mg}$ al mes por seis meses. El cuadro clínico se resolvió en forma adecuada, sin recidivas hasta el momento.

\section{CONCLUSIÓN}

La pitiriasis versicolor variedad acrómica o vitiligoide constituye un reto diagnóstico para el dermatólogo debido a la baja frecuencia con que se presenta; por lo cual, ante un caso como éste, desde un inicio se deben plantear los diagnósticos diferenciales, tales como la hipomelanosis

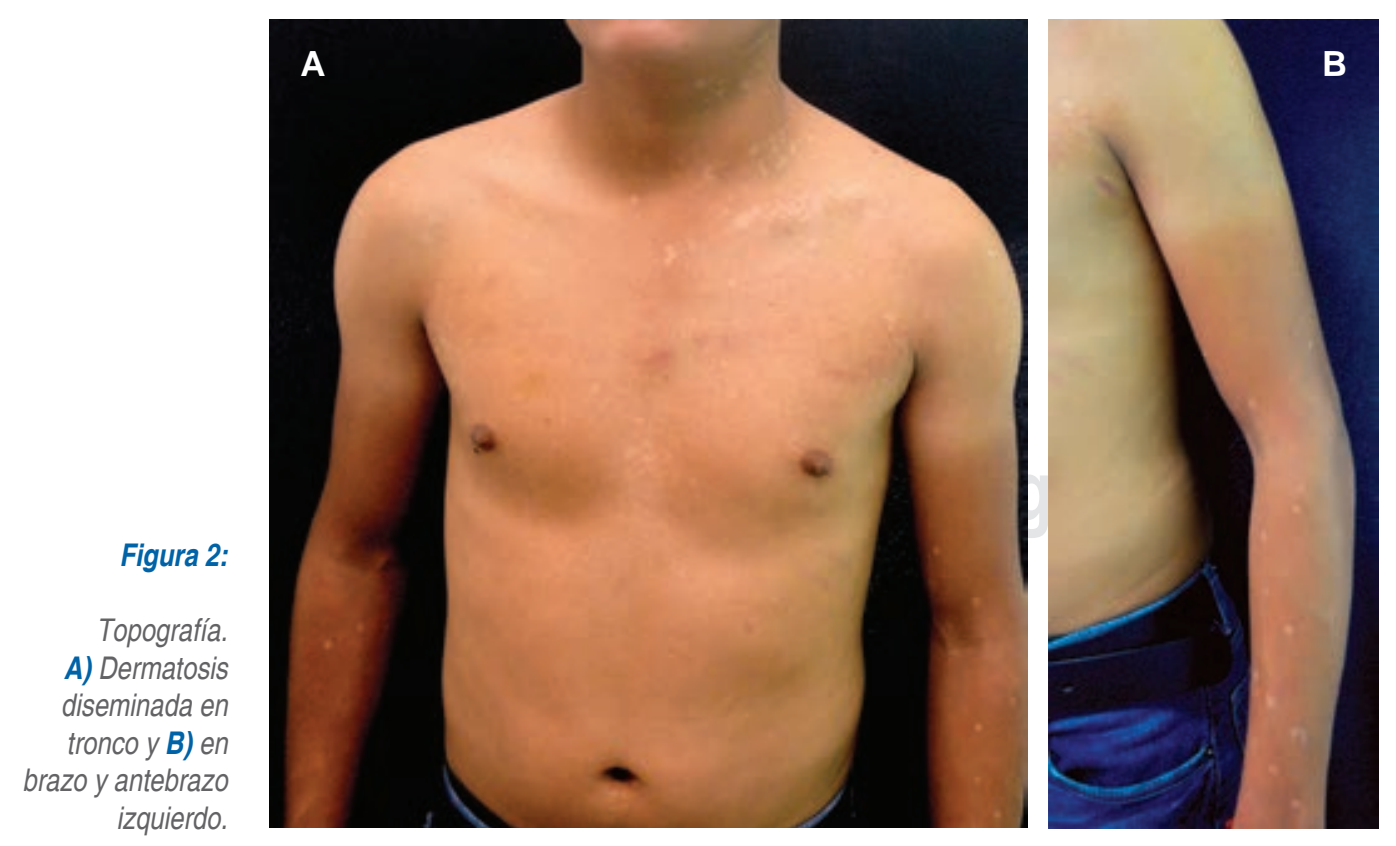




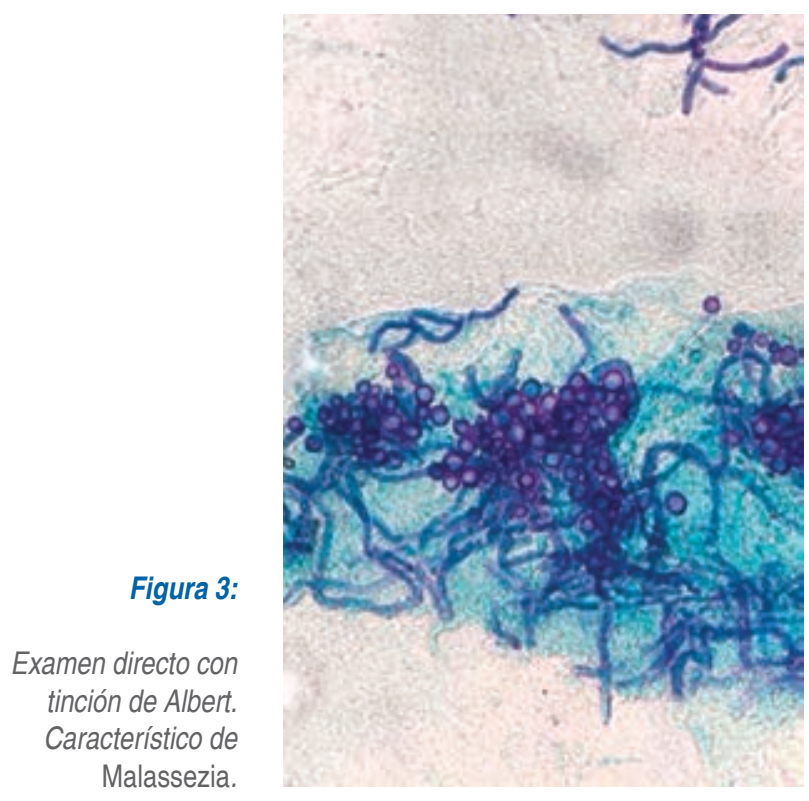

macular progresiva idiopática, y en especial con el vitíligo, puesto que este último genera ansiedad extrema debido al aspecto cosmético y al estigma social.

Ante la sospecha clínica es importante confirmar el diagnóstico a través de un examen micológico directo con cinta adhesiva. Debe explicarse al paciente en forma detallada los diferentes aspectos de esta micosis, prescribir el tratamiento adecuado, e indicar medidas generales para evitar las recidivas. El caso motivo de esta publicación es muy interesante, por lo infrecuente de la variedad vitiligoide y la topografía en pliegues antecubitales, y que en la recurrencia desarrolló la forma eritematosa dermatofitoide. Se decidió incrementar la dosis de itraconazol, con administración profiláctica mensual, se obtuvo remisión del cuadro clínico hasta el momento.

\section{REFERENCIAS}

1. Lorch JM, Palmer JM, Vanderwolf KJ, Schmidt KZ, Verant ML, Weller TJ et al. Malassezia vespertilionis sp. nov.: a new cold-tolerant species of yeast isolated from bats. Persoonia. 2018; 41: 56-70.

2. Guillot J, Gueho E, Lesourd M, Midgley G, Chevrier G, Dupont B. Identification of Malassezia species. A practical approach. J Mycol Med. 1996; 6: 103-110.

3. Cabañes FJ, Coutinho SD, Puig L, Bragulat MR, Castellá G. New lipid-dependent Malassezia species from parrots. Rev Iberoam Micol. 2016; 33: 92-99.

4. Böhmová E, Conková E, Sihelská Z, Harcárová M. Diagnostics of Malassezia species: a review. Folia Veterinaria. 2018; 62: 19-29.

5. Gupta AK, Batra R, Bluhm R, Boekhout T, Dawson TL Jr. Skin diseases associated with Malassezia species. J Am Acad Dermatol. 2004; 51 : 785-798.
6. Morales BME, Padilla DMC, Martínez MJA. Pitiriasis versicolor variedad hipercrómica. Comunicación de un caso. Rev Cent Dermatol Pascua. 2007; 16: 93-95.

7. Kaushik N, Pujalte GG, Reese ST. Superficial fungal infections. Prim Care. 2015; 42: 501-516.

8. Padilla-Desgarennes MC. Pitiriasis versicolor. Dermatología Rev Mex. 2005; 49: 157-167.

9. Karray M, McKinney WP. Tinea versicolor [Updated 2021 Aug 11] In: StatPearls [Internet]. Treasure Island (FL): StatPearls Publishing; 2022. Available in: https://www.ncbi.nlm.nih.gov/books/NBK482500/

10. Schwartz RA. Superficial fungal infections. Lancet. 2004; 364: 11731182.

11. Giusiano Gustavo E. Malassezia: Estado del conocimiento y perspectivas en su estudio. Rev Argent Microbiol. 2006; 38: 41-48.

12. Santamaría GV, Alvarado DA. Flora cutánea como protección y barrera de la piel normal. Rev Cent Dermatol Pascua. 2002; 11: 18-21.

13. Sparber F, LeibundGut-Landmann S. Host responses to Malassezia spp. in the mammalian skin. Front Immunol. 2017; 8: 1614.

14. Prohic A, Jovovic Sadikovic T, Krupalija-Fazlic M, KuskunovicVlahovljak S. Malassezia species in healthy skin and in dermatological conditions. Int J Dermatol. 2016; 5: 494-504.

15. Isa Isa $R$, Cruz $A C$, Arenas $R$, Duarte $Y$, Linares $C M$, Bogaert $H$. Pitiriasis versicolor en niños. Estudio epidemiológico y micológico de 797 casos estudiados en la República Dominicana. Med Cutan Iber Lat Am. 2002; 30: 5-8.

16. Ramadán S, Sortino M, Bulacio L, Marozzi ML, López C, Ramos L. Prevalence of Malassezia species in patients with pityriasis versicolor in Rosario, Argentina. Rev Iberoam Micol. 2012; 29: 14-19.

17. Torres $\mathrm{E}$, Arenas $\mathrm{R}$, Atoche-Diéguez $\mathrm{C}$. Infecciones causadas por el género Malassezia. Med Cutan Iber Lat Am. 2008; 36: 265-284.

18. Varada S, Dabade T, Loo DS. Uncommon presentations of tinea versicolor. Dermatol Pract Concept. 2014; 4: 93-96.

19. Mollet I, Ongenae K, Naeyaert JM. Origin, clinical presentation, and diagnosis of hypomelanotic skin disorders. Dermatol Clin. 2007; 25: 363-371.

20. Thoma W, Kramer HJ, Mayser P. Pityriasis versicolor alba. J Eur Acad Dermatol Venereol. 2005; 19: 147-152.

21. Kallini JR, Riaz F, Khachemoune A. Tinea versicolor in dark-skinned individuals. Int J Dermatol. 2014; 53: 137-141.

22. Rivard SC. Pityriasis versicolor: avoiding pitfalls in disease diagnosis and therapy. Mil Med. 2013; 178: 904-906.

23. Huang WW, Tharp MD. A case of tinea versicolor of the eyelids. Pediatr Dermatol. 2013; 30: e242-e243.

24. Errichetti E, Stinco G. Dermoscopy in general dermatology: a practical overview. Dermatol Ther (Heidelb). 2016; 6: 471-507.

25. Bonifaz A. Micología médica básica. 4a ed. México: McGraw-Hill; 2012. pp. 135-151.

26. Arenas R, Padilla Desgarennes MC, Bonifaz A, Mayorga Rodríguez J. Pitiriasis versicolor y otras entidades relacionadas con Malassezia. PAC Dermatología 3. 2014; 7: 376-382.

\section{Correspondencia:}

Dra. María del Carmen Padilla-Desgarennes

Dr. Vértiz Núm. 464, esq. Eje 3 Sur,

Col. Buenos Aires, 06780,

Alcaldía Cuauhtémoc, CDMX.

Tel: 55 5519-6351

E-mail: mcpadillad@prodigy.net.mx 